\title{
Cargas de trabalho na Estratégia Saúde da Família: interfaces com o desgaste dos profissionais de enfermagem*
}

\author{
Workloads in the Family Health Strategy: interfaces with the exhaustion of nursing professionals \\ Cargas de trabajo en la Estrategia de Salud Familiar: interfaces \\ con el desgaste de los profesionales de la enfermería
}

Como citar este artigo:

Mendes M, Trindade LL, Pires DEP, Biff D, Martins MMFPS, Vendruscolo C. Workloads in the Family Health Strategy: interfaces with the exhaustion of nursing professionals. Rev Esc Enferm USP. 2020;54:e03622. doi: https://doi.org/10.1590/S1980-220X2019005003622

\section{Mariana Mendes ${ }^{1}$ \\ Letícia de Lima Trindade ${ }^{1}$ \\ Denise Elvira Pires de Pires $^{2}$ \\ Daiane Biff ${ }^{2}$ \\ Maria Manuela Ferreira Pereira da} Silva Martins ${ }^{3}$

\section{(D) Carine Vendruscolo ${ }^{4}$}

* Extraído da dissertação: "Processo de trabalho da enfermagem na Estratégia Saúde da Família e as cargas de trabalho no Brasil", Programa de PósGraduação em Ciências da Saúde, Universidade

Comunitária da Região de Chapecó, 2018.

${ }^{1}$ Universidade Comunitária da Região de Chapecó, Programa de Pós-graduação em Ciências da Saúde, Chapecó, SC, Brasil.

${ }^{2}$ Universidade Federal de Santa Catarina, Programa de Pós-Graduação em

Enfermagem, Florianópolis, SC, Brasil.

${ }^{3}$ Centro de Investigação em Tecnologia

e Serviços de Saúde, Escola Superior de

Enfermagem do Porto, Porto, Portugal.

${ }^{4}$ Universidade do Estado de Santa

Catarina, Florianópolis, SC, Brasil.

\begin{abstract}
Objective: To identify the workloads of nursing teams working in the Family Health Strategy in the five regions of Brazil and their interfaces with their feelings of exhaustion. Method: Qualitative study with nursing professionals from 20 Family Health Units, from eight cities in the five regions of the country. Data were collected through interviews, observation and analyzed according to the precepts of thematic analysis and the theoretical framework of workloads. Results: A total of 79 professionals participated in the study. Workloads were related to structural and managerial problems. Workloads that stood out were psychological workload, due to excessive demands and staff shortages; physiological workload, due to the overload of activities that generate physical pain and exhaustion; physical and mechanical workload, due to inadequacies in the work environment and equipment; biological workload, due to the presence of microorganisms; and chemical workload, due to exposure to dust and smoke. Conclusion: The sources that increase workloads are similar in the five regions, indicating that there is a strong influence of psychological and physiological loads on exhaustion among workers. However, workloads are reduced by dividing and planning team actions and by the affinity with the assistance model.
\end{abstract}

\section{DESCRIPTORS}

Primary Care Nursing; Workload; Nursing, Team; Occupational Health; Family Health Strategy. 


\section{INTRODUÇÃO}

No Brasil, a Atenção Primária à Saúde (APS) é operacionalizada pela Estratégia Saúde da Família (ESF). Essa modalidade assistencial é prevista na Política Nacional de Atenção Básica $(\mathrm{PNAB})^{(1)}$ como estratégia fundamental para a melhoria da assistência à saúde. Desde a implantação das equipes de Saúde da Família (SF), evidencia-se melhora no acesso aos serviços e nos indicadores de saúde, como redução na taxa da morbimortalidade infantil e diminuição no número de internações hospitalares potencialmente evitáveis, dentre outros ${ }^{(2)}$.

$\mathrm{Na}$ ESF, está prevista a realização do trabalho por equipes de SF compostas por, no mínimo, um médico e um enfermeiro, preferencialmente especialistas em saúde da família, um técnico ou auxiliar de enfermagem e agentes comunitários de saúde (ACS), sendo que cada equipe se responsabiliza por 2.000 a 3.500 pessoas $^{(1)}$. Na APS, a enfermagem desempenha papel central, contribuindo para melhorar os determinantes sociais de saúde-doença, especialmente no atendimento às pessoas em situação de vulnerabilidade e na promoção do acesso aos serviços de saúde ${ }^{(3-7)}$.

O trabalho da enfermagem é complexo e envolve diversos aspectos do fazer típico da profissão, os quais podem ser geradores de cargas de trabalho. No contexto da APS, estão presentes inúmeros desafios típicos do modelo assistencial, demandando dos profissionais de enfermagem um fazer criativo, com ênfase na resolutividade, na integralidade, na atenção individual e coletiva e para além do ambiente físico das unidades assistenciais, o que acrescenta complexidade e diversidade às cargas de trabalho e promove o acesso universal à saúde ${ }^{(8)}$.

Para apreender de que forma se desenvolve o processo de trabalho da enfermagem no cenário da ESF, bem como as cargas de trabalho existentes, é necessário compreender a dimensão técnica e social que o envolve e o modo como os processos corporais podem refletir a relação entre trabalhador, organização e divisão do trabalho ${ }^{(9)}$. As cargas de trabalho interatuam entre si e com o corpo de quem realiza o trabalho, não atuando isoladamente, mas em combinação umas com as outras. Elas determinam a condição pela qual o trabalhador enfrenta a lógica global do processo de trabalho, caracterizada como física, química, biológica, mecânica, fisiológica e psíquica ${ }^{(9)}$.

Nesse sentido, o estudo tem por objetivo identificar as cargas de trabalho da equipe de enfermagem e suas interfaces com o desgaste desses profissionais que atuam em equipe da SF nas diferentes regiões do Brasil. Para tanto, visa responder os seguintes questionamentos: a quais cargas de trabalho estão expostos os profissionais da enfermagem que atuam na SF nas cinco regiões do Brasil? Quais são as interfaces das cargas de trabalho no adoecimento desses profissionais?

\section{MÉTODO}

\section{TIPO DE ESTUDO}

Trata-se de estudo qualitativo com dados obtidos mediante triangulação de instrumentos, entrevistas semiestruturadas e observação, sendo orientado pela teoria da carga de trabalho de Laurell e Noriega ${ }^{(9)}$. Para realização do estudo, foram envolvidos pesquisadores de seis universidades brasileiras, das cinco regiões do país, que participaram de alinhamento teórico e metodológico periódico em seminários nacionais realizados na região Sul.

\section{Cenário}

A escolha do cenário seguiu os seguintes critérios de inclusão: Unidades Básicas de Saúde (UBS) que atuavam somente no modelo assistencial da ESF e que dispunham de equipes com a composição mínima prescrita na Política Nacional da Atenção Básica (PNAB); equipes consideradas de boa qualidade, segundo indicação de gestores e lideranças profissionais em cada região, associando-se dados publicados nos resultados do Programa Nacional de Melhoria do Acesso e da Qualidade da Atenção Básica (PMAQ-AB) ${ }^{(10)}$; no mínimo um município por região do país.

Para a escolha dos municípios, participantes foram associados aos critérios de intencionalidade e conveniência, considerando a possibilidade de acesso às diversas regiões geográficas do país, sendo os seguintes: Araranguá e Florianópolis (Sul); Brasília (Centro-Oeste); Rio de Janeiro (Sudeste); Manaus, Belém e Nova Olinda do Norte (Norte); e Natal (Nordeste). O número de UBS participantes totalizou 21, sendo sete unidades no Sul, cinco no Centro-Oeste, duas no Sudeste, três no Norte e quatro no Nordeste.

Para composição da população do estudo, utilizaram-se como critérios de inclusão ser da categoria de enfermagem e atuar há pelo menos um ano na ESF. Como critério de exclusão, definiu-se: estar afastado por qualquer motivo no momento da coleta de dados.

A população de estudo totalizou 79 participantes, dos quais 45 eram enfermeiros, 30 técnicos de enfermagem e quatro auxiliares de enfermagem. O quantitativo de profissionais e equipes envolvidos foi considerado suficiente pelo critério de saturação de dados, evidenciado quando não foram mais encontradas novas explicações, interpretações ou descrições do fenômeno estudado, sendo esse critério avaliado coletivamente nos seminários metodológicos que contaram com a presença dos pesquisadores que coordenaram o processo de coleta de dados nas cinco regiões do país.

\section{Colleta de dados}

Para a coleta de dados, foram realizadas entrevistas com participantes, buscando-se identificar/caracterizar as informações relativas ao trabalho da enfermagem na ESF, a organização e divisão do trabalho da equipe, a caracterização do modelo assistencial ESF, bem como a sua influência na carga de trabalho da categoria. As entrevistas foram realizadas no local de trabalho, mediante agendamento prévio, gravadas e posteriormente transcritas na íntegra.

Também foram realizadas observações de, no mínimo, um dia de trabalho em cada região/município/UBS, mediante roteiro, sendo que os dados foram registrados em diário de campo. A observação teve por finalidade apreender as formas de divisão, organização e gestão do trabalho na enfermagem e na SF. 
Os roteiros de entrevista e observação foram elaborados nos seminários nacionais, buscando-se a padronização de conteúdo e conduta na coleta e análise dos dados. Previamente à coleta de dados, os roteiros foram testados, com realização dos ajustes necessários.

Com vistas a atender o objetivo da pesquisa e possibilitar um aprofundamento da compreensão do fenômeno estudado, associou-se ao recurso de triangulação de instrumentos o envolvimento das diversas regiões do Brasil. Assim, para viabilizar a coleta de dados em um país com as dimensões continentais do Brasil, foi necessário construir um banco de dados obtidos no período de 2013 a 2018.

\section{AnÁlise E TRATAMENTO DOS DADOS}

Para a análise, foram utilizados os recursos do software Atlas.ti 7.0 (Qualitative Research and Solutions) e os preceitos da Análise de Conteúdo Temática, interpretando os achados com base no referencial teórico escolhido.

Na pré-análise, foi criada, no software, uma unidade de armazenamento (Hermeneutic Unit), na qual foram inseridos os dados obtidos nas entrevistas e observações (Primary Documents). Na fase de exploração do material, foram selecionadas unidades de significado (Quotations) às quais se atribuíram códigos (Codes) que, posteriormente, foram agrupados em Families passíveis de visualização em redes (Networks). No processo de interpretação, foram feitas as articulações entre quotations, codes e families, possibilitando a organização de temas e elaboração das categorias de análise. Nesse processo, utilizou-se, principalmente, o recurso da visualização em redes (Networks), que pode ser extraída do software e apresentada nos resultados.

\section{AsPeCtos ÉTICOS}

Todos os preceitos éticos relativos à pesquisa envolvendo seres humanos e aos dispositivos constantes nas Resoluções no 466/2012, do Conselho Nacional de Saúde, foram respeitados. A pesquisa foi aprovada pelo Comitê de Ética em Pesquisa da Universidade do Estado de Santa Catarina (Pareceres no 366.844/2010 e no 1.933.348/2017). Os participantes receberam informações sobre os procedimentos éticos e assinaram o Termo de Consentimento Livre e Esclarecido. Garantiram-se o sigilo e o anonimato mediante a codificação dos participantes, utilizando-se letras iniciais de cada categoria: letra E (Enfermeiro), Te (Técnico de Enfermagem), Ae (Auxiliar de Enfermagem). Tais códigos foram seguidos da(s) letra(s) que identifica $(\mathrm{m})$ cada região - S (Sul), CO (Centro-Oeste), N (Norte), SE (Sudeste), NE (Nordeste) - e do número sequencial (Por exemplo: EN1, EN2, TES3, TECO4, sucessivamente). Os trechos de observação foram apresentados com a sigla OBS, seguida da região.

\section{RESULTADOS}

\section{Perfil sociolaboral da força de trabalho da ENFERMAGEM NAS EQUIPES DE SF PESQUISADAS}

O perfil sociolaboral dos participantes encontra-se na Tabela 1.

Tabela 1 - Distribuição dos participantes quanto ao perfil sociolaboral - Florianópolis, SC, Brasil, 2018.

\begin{tabular}{|c|c|c|c|c|c|}
\hline Variáveis & Descrição das variáveis & $\begin{array}{l}\text { Número de } \\
\text { Enfermeiros }\end{array}$ & $\begin{array}{l}\text { Número de Técnicos } \\
\text { de Enfermagem }\end{array}$ & $\begin{array}{l}\text { Número de Auxiliares } \\
\text { de Enfermagem }\end{array}$ & Total \\
\hline \multirow{2}{*}{ Sexo } & Feminino & 41 & 27 & 4 & 72 \\
\hline & Masculino & 4 & 3 & - & 7 \\
\hline \multirow{5}{*}{ Idade } & $<20$ anos & - & - & - & - \\
\hline & 20 a 40 anos & 26 & 16 & - & 42 \\
\hline & 41 a 50 anos & 10 & 9 & 1 & 20 \\
\hline & 51 a 60 & 8 & 4 & 2 & 14 \\
\hline & $>60$ anos & 1 & 1 & 1 & 3 \\
\hline \multirow{4}{*}{ Escolaridade } & Ensino médio & - & 28 & 4 & 32 \\
\hline & Graduação & 3 & 2 & - & 5 \\
\hline & Especialização/residência & 37 & - & - & 37 \\
\hline & Mestrado & 5 & - & - & 5 \\
\hline \multirow{2}{*}{ Tempo experiência profissional } & 1 a 5 anos & 8 & 11 & - & 19 \\
\hline & $>5$ anos & 37 & 19 & 4 & 60 \\
\hline \multirow{3}{*}{ Tempo experiência na ESF } & 1 ano & - & 2 & - & 2 \\
\hline & 1 a 5 anos & 18 & 17 & - & 35 \\
\hline & $>5$ anos & 27 & 11 & 4 & 42 \\
\hline \multirow{2}{*}{ Tipo de contratação } & Concursado/estatutário & 29 & 15 & 3 & 47 \\
\hline & Temporário e celetista & 16 & 15 & 1 & 32 \\
\hline \multirow{3}{*}{ Jornada de trabalho (semanal) } & $<40$ horas & 4 & 1 & - & 5 \\
\hline & 40 horas & 26 & 21 & 3 & 50 \\
\hline & $>40$ horas & 15 & 8 & 1 & 24 \\
\hline \multirow{2}{*}{ Outro emprego } & Possui & 14 & 9 & 1 & 24 \\
\hline & Não possui & 31 & 21 & 3 & 55 \\
\hline
\end{tabular}




\section{Cargas de traballho Presentes no PROCESSO DE}

\section{TRABALLHO DA ENFERMAGEM NAS EQUIPES DE SF PESQUISADAS}

Nessa categoria de análise, estão apresentados os aspectos/elementos presentes no processo de trabalho da enfermagem da ESF que se constituem de fontes de aumento das cargas de trabalho, podendo gerar desgaste ou adoecimento. As cargas podem atuar de modo isolado ou se inter-relacionar, sendo que um aspecto/elemento presente no processo de trabalho pode gerar mais de um tipo de carga. O Quadro 1 mostra o tipo de cargas, as fontes de aumento e as evidências identificadas nas entrevistas e observações.

Quadro 1 - Tipos de carga e fontes de aumento das cargas de trabalho na equipe de enfermagem na ESF - Florianópolis, SC, Brasil, 2018.

\begin{tabular}{|c|c|c|}
\hline Tipo de CT & Fontes de aumento das CT & Trechos de falas e observações que evidenciam as CT \\
\hline \multirow{4}{*}{ Cargas Psíquicas } & $\begin{array}{l}\text { Excesso de demanda programada e } \\
\text { espontânea. }\end{array}$ & $\begin{array}{l}\text { A demanda é muito grande, porque a gente não tem profissional suficiente na rede. } \\
\text { Na minha área, a gente tem nove mil habitantes. É muito longe do preconizado (ES3). } \\
\text { A pressão psicológica referente à quantidade de pessoas a serem atendidas, espera } \\
\text { por atendimento, era perceptível na inquietação dos profissionais, no momento da } \\
\text { entrevista (OBS SUDESTE). }\end{array}$ \\
\hline & $\begin{array}{l}\text { O déficit de funcionários inclui } \\
\text { número insuficiente para atender os } \\
\text { usuários; as licenças e afastamentos } \\
\text { por doença e/ou atestado. }\end{array}$ & $\begin{array}{l}\text { Eu acho que pode melhorar, mas para isso você precisa de recursos materiais, } \\
\text { recursos humanos também, porque a gente trabalha com pouquíssimos profissionais e } \\
\text { faltas (...) sempre tem falta de profissionais e um profissional tendo que cobrir outro e } \\
\text { ficar com uma sobrecarga (ECO5). }\end{array}$ \\
\hline & Insatisfação do usuário. & $\begin{array}{l}\text { Você tem um monte de papelada que faz com que você perca tempo (...) } \\
\text { Muitas vezes, a pessoa fica no corredor e só escuto reclamação (...) não sabem } \\
\text { que é questão burocrática, é muita coisa para anotar, que faz com que o serviço } \\
\text { demore (AeNE1). }\end{array}$ \\
\hline & Sobrecarga de atividades. & $\begin{array}{l}\text { Então o técnico tem muitas atribuições (TeSE3). } \\
\text { Nós técnicos não somos só técnicos. Nós somos porteiro, telefonistas, digitador, } \\
\text { farmacêutico (TeN6). }\end{array}$ \\
\hline \multirow{4}{*}{ Cargas Fisiológicas } & $\begin{array}{l}\text { Excesso de demanda programada e } \\
\text { espontânea. }\end{array}$ & $\begin{array}{l}\text { Ter que realizar } 98 \text { visitas domiciliares, para um técnico, tenho que estar na sala de } \\
\text { curativo, na sala de medicação, na dispensação de medicamentos, na triagem dos } \\
\text { pacientes, no agendamento das consultas, fazendo medicação. Então, o profissional } \\
\text { tem consciência que faz um serviço bem feito, assim como eu, sai do trabalho } \\
\text { muito cansada, por isso não penso em ter outro trabalho. Não consigo fazer outras } \\
\text { atividades. Quando chego em casa, já chego cansada (TeCO5). } \\
\text { Aqui, o volume de pessoas é muito grande. Agora, é atípico eu estar aqui conversando } \\
\text { com você porque eu não tenho nem tempo para ir ao banheiro (...) (TeS2). }\end{array}$ \\
\hline & Sobrecarga de atividades. & $\begin{array}{l}\text { A sobrecarga de serviço é muito prejudicial para a saúde da gente. Já vi muita gente } \\
\text { adoecendo, a própria médica da equipe, sem contar as lesões. Esses dias mesmo, eu } \\
\text { estava que não conseguia nem levantar os braços. Não conseguia movimentar a mão } \\
\text { (...) a sobrecarga de trabalho me prejudicou bastante. Às vezes, sou privada de tomar } \\
\text { água, de ir ao banheiro, de sentar alguns minutos para descansar (TeCO5). }\end{array}$ \\
\hline & $\begin{array}{l}\text { O déficit de funcionários inclui } \\
\text { número insuficiente para atender os } \\
\text { usuários; as licenças e afastamentos } \\
\text { por doença e/ou atestado. }\end{array}$ & $\begin{array}{l}\text { Acho que a sobrecarga de trabalho é pela falta de profissional. A gente se estressa } \\
\text { muito (TeCO1). } \\
\text { Somos apenas duas técnicas de enfermagem na nossa equipe (...). Um funcionário só } \\
\text { para a farmácia [diminuiria] essa sobrecarga (...), aí, eu poderia estar ajudando mais a } \\
\text { comunidade, poderia estar fazendo mais visitas (TeCO3). }\end{array}$ \\
\hline & Jornada de trabalho excessiva. & $\begin{array}{l}\text { (...) nem almoço a gente tem direito aqui (...) a gente compra o almoço, não tem } \\
\text { um repouso, não tem nada. Se fosse pelo menos unas } 30 \text { horas, como a gente está } \\
\text { tentando, talvez melhorasse (TeNE1). }\end{array}$ \\
\hline Cargas Biológicas & $\begin{array}{l}\text { Exposição a agentes biológicos, como } \\
\text { bactérias e vírus, pelo manuseio } \\
\text { de imunobiológicos e realização } \\
\text { de procedimentos envolvendo } \\
\text { contaminação; contato com doenças } \\
\text { infectocontagiosas; e presença de } \\
\text { leveduras (mofo) em decorrência de } \\
\text { estrutura física inadequada. }\end{array}$ & $\begin{array}{l}\text { Conheço uma enfermeira que pegou Hepatite A, dois ACS que pegaram tuberculose e } \\
\text { uma técnica que teve acidente com perfurocortante (EN2). } \\
\text { Existe uma sala sem condições mínimas, não tem pia dentro dessa sala, não tem } \\
\text { descarpack para dispensação adequada de material perfuro-cortante. Foi improvisado } \\
\text { uma lata de "leite em pó" para descarte das agulhas (OBS CENTRO-OESTE). }\end{array}$ \\
\hline Cargas Físicas & $\begin{array}{l}\text { Exposição ao calor ou frio intensos e } \\
\text { à umidade está intimamente ligada às } \\
\text { condições ambientais e de estrutura } \\
\text { física das UBS. }\end{array}$ & $\begin{array}{l}\text { Aqui é uma casa alugada, adaptada (...) a gente teve um problema muito sério, a casa } \\
\text { com muita infiltração, muito mofo, muita goteira e quando chovia a gente não podia } \\
\text { ficar na Unidade (ENE6). } \\
\text { (...) sem ar-condicionado, não tem janela, não tem ventilação nenhuma. Então, acaba } \\
\text { se sentindo mal (...) tenho queda de pressão, fico irritada (TeSE3). }\end{array}$ \\
\hline Cargas Mecânicas & $\begin{array}{l}\text { Equipamentos inadequados para uso } \\
\text { na assistência (sem manutenção), falta } \\
\text { de móveis e/ou impróprios. }\end{array}$ & $\begin{array}{l}\text { As infiltrações, quando chove, molha. Podemos escorregar e cair. É a segurança que } \\
\text { não temos. Além disso, a escada, porque a gente fica subindo e descendo, podendo } \\
\text { até cair, e a própria entrada da Unidade é difícil, pois a vala é aberta e não tem um } \\
\text { tubo do esgoto (EN2). } \\
\text { Não possui equipamentos em condições para assistência em saúde. Evidenciou- } \\
\text { se instrumentos e equipamentos essenciais para prática da assistência, muitos } \\
\text { em péssimas condições, como armários calçados por pedras, cadeiras de ferro } \\
\text { enferrujadas, não obedecendo aos padrões ergonômicos (OBS NORDESTE). }\end{array}$ \\
\hline Cargas Químicas & Exposição a poeiras e fumaças. & $\begin{array}{l}\text { Nessa região da [UBS], o ar bem pesado, devido às fábricas de cimento que têm } \\
\text { instaladas aqui. Isso acaba refletindo na saúde respiratória de alguns colegas (ECO9). }\end{array}$ \\
\hline
\end{tabular}


As cargas de trabalho psíquicas estão presentes no cotidiano da enfermagem que trabalha na ESF, sendo as mais significativas. Além disso, as fontes que as elevam estão fortemente relacionadas ao excesso de demanda e às falhas na gestão. De modo semelhante, as fontes das cargas de trabalho fisiológicas são o acúmulo de atividades assistenciais e administrativas para os enfermeiros e a sobrecarga de atividades para as demais categorias de enfermagem. Nas cargas de trabalho biológicas, predomina o contato com micro-organismos entre técnicos e auxiliares, enquanto as químicas foram relacionadas ao ambiente externo da unidade. As cargas de trabalho físicas e mecânicas estiveram associadas ao ambiente de trabalho e foram identificadas, predominantemente, pelos técnicos de enfermagem.
Não houve diferença entre as regiões no que tange às fontes de aumento das cargas de trabalho e tipos de cargas, com exceção das cargas químicas mencionadas somente na região Centro-Oeste, inferindo-se que as dificuldades e os desafios são muito semelhantes.

\section{FONTES DE REDUÇÃO DAS CARGAS DE TRABALHO DA ENFERMAGEM NAS EQUIPES DE SF PESQUISADAS}

Nessa categoria, estão identificados aspectos/elementos do processo de trabalho da enfermagem da ESF que constituem fontes de redução das cargas de trabalho, contribuindo como protetores em relação ao desgaste e adoecimento, conforme está ilustrado na Figura 1.

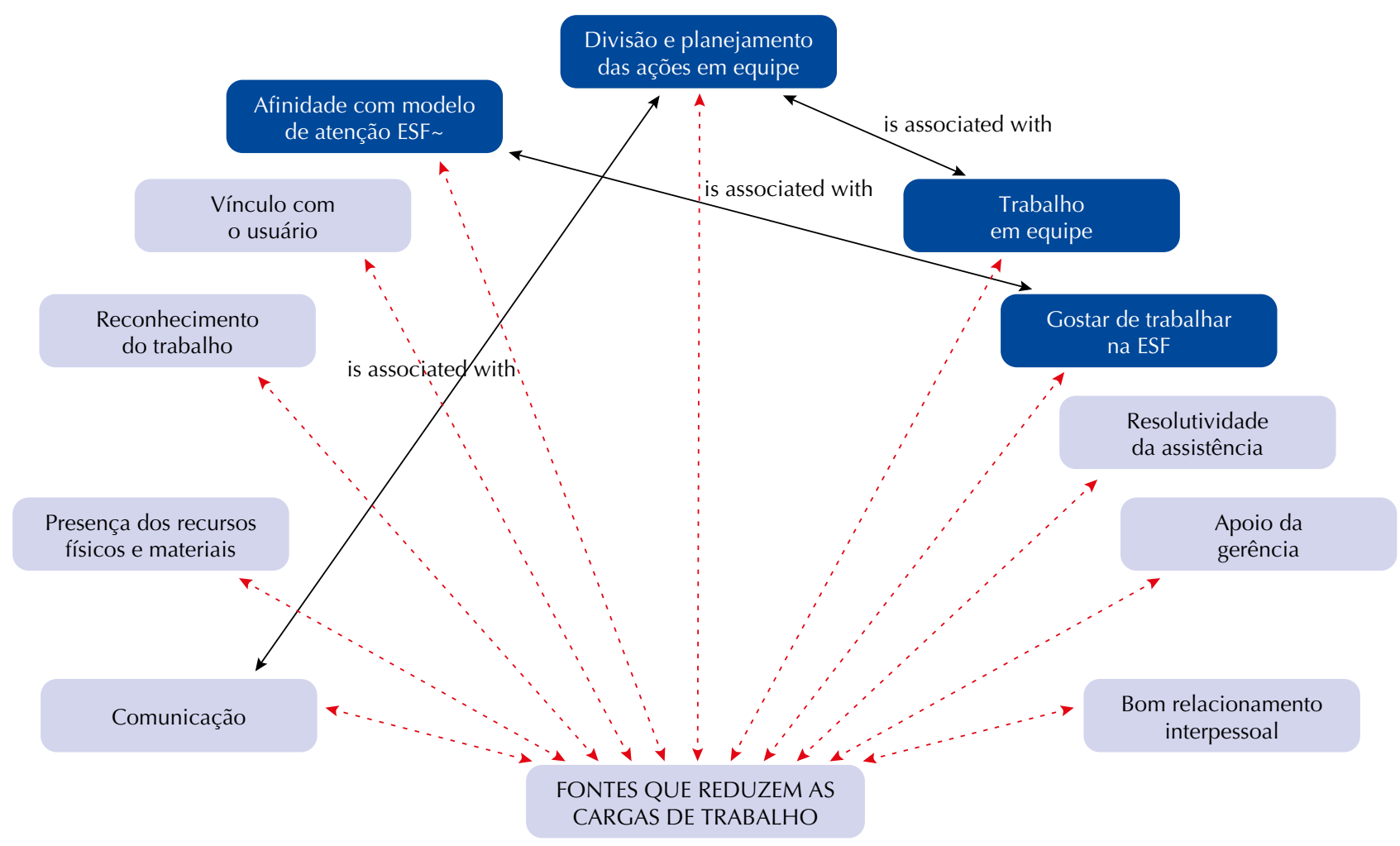

Legenda: Azul escuro, mais citado pelos participantes.

Figura 1 - Fontes que reduzem as cargas de trabalho da equipe de enfermagem na ESF - Florianópolis, SC, Brasil, 2018.

A divisão e o planejamento das ações em equipe constituem a principal fonte de redução das cargas de trabalho da enfermagem na ESF, o que está associado ao trabalho em equipe e à boa comunicação entre os diversos componentes da equipe.

A diferença para a Unidade funcionar é a equipe toda (...) se todo o mundo falar a mesma língua pode ter 10 mil habitantes, vai funcionar (...) Isso é o que faz diferença e quando a equipe trabalha unida por mais que não tenha o necessário (TeS4).

A afinidade com o modelo da ESF está relacionada ao prazer em trabalhar na Estratégia e constitui importante redutor das cargas de trabalho.
(...) quem trabalha como enfermeiro, como coordenador de uma equipe de Saúde da Família, tem que ter uma (...) motivação interior (...) Algo que faça você querer fazer o seu trabalho diário, mesmo quando falta alguma coisa (...) que te motive a acolher um paciente, a conversar, a conhecer a comunidade (ECO9).

O vínculo com o usuário foi identificado como prescrição da PNAB que, se efetivado, contribuiria para a melhoria dos cuidados prestados.

$\mathrm{Na}$ Unidade, todos são acolhedores. A escuta qualificada deve ser feita não só por uma pessoa em um certo horário (...) todo o mundo tem esse poder, de ouvir, de buscar uma resolutividade dentro das possibilidades de cada um. Eu vejo isso como muito 
positivo na minha unidade. Essa busca do acolbimento, da bumanização (ENE7).

Além disso, quando valorizados e reconhecidos pela gestão/gerência, sentem-se amparados e com suas necessidades acolhidas, fato mencionado preponderantemente por enfermeiros.

(...) eu tenho o apoio da minha equipe, eu tenho o apoio da gerência. A gerência sempre, assim, entende e tenta também combater essa insatisfação da gente (EN5).

Para a equipe de enfermagem, a disponibilidade de recursos físicos adequados amplia o potencial de atendimento e resolutividade da equipe de $\mathrm{SF}$.

Eu tenho possibilidades de atuação e ampliação da minha resolutividade enquanto profissional de saúde muito maior se comparada às outras unidades de saúde no Brasil (...). Muitas unidades não têm nem prontuário eletrônico. Eu tenho sistema que é informatizado, eu consigo fazer ultrassom, eu consigo fazer raio- $X$, eu consigo fazer exames de sangue. Eu tenho uma série de facilidades que ampliam a minha resolutividade (ESE4).

\section{DISCUSSÃO}

No que diz respeito ao perfil dos profissionais de enfermagem, no cenário de APS estudado, os resultados foram semelhantes ao descrito em estudo realizado no Brasil ${ }^{(11)} \mathrm{e}$ no âmbito internacional ${ }^{(12)}$. Verificou-se predomínio da força de trabalho feminina e de jornada de trabalho de 40 horas semanais ou superior, sendo que 30,37\% têm duplo emprego.

Em relação à escolaridade, identificou-se que a grande maioria dos enfermeiros possui formação na pós-graduação, com especialização ou mestrado. Estudos realizados na área hospitalar mostram que a qualificação da força de trabalho da enfermagem impacta positivamente na qualidade da assistência ${ }^{(13)}$.

Em relação ao tipo de contratação, observou-se que $50 \%$ dos técnicos de enfermagem são contratados na modalidade temporária ou pela Consolidação das Leis do Trabalho (CLT), especialmente nas regiões Sudeste e Sul. Pesquisadores ${ }^{(7,12,14)}$ sinalizam para a importância de relações estáveis de trabalho, as quais favorecem os investimentos na qualificação dos profissionais, reduzem a rotatividade e fortalecem o vínculo entre profissional e usuários. Isso gera ainda mais preocupação frente à aprovação da Lei da Terceirização, $n^{\circ} 13.429 / 2017$, que amplia as possibilidades de contratos terceirizados e jornadas em regime especial.

A partir da compreensão da lógica global do processo de trabalho da enfermagem na ESF, evidenciou-se que os diversos tipos de cargas de trabalho estão presentes em todas as regiões, com exceção das cargas químicas. As cargas de trabalho causam impactos no corpo do trabalhador, podendo gerar desgaste ou adoecimento. Cabe considerar, ainda, que as cargas de trabalho interatuam entre si e o corpo do trabalhador, sendo que a reprodução de processos alienantes, parcelados e que limitam as capacidades dos trabalhadores alteram seus processos biopsíquicos ${ }^{(9)}$.

As cargas psíquicas são predominantes na equipe de enfermagem e se relacionaram a problemas estruturais e gerenciais, sendo o excesso de demanda, em associação ao déficit de pessoal e à sobrecarga de trabalho, a principal fonte. Vale destacar que problemas estruturais, quantitativos inadequados de pessoas para assistência segura, problemas na organização do trabalho e na gestão na APS repercutem no desgaste dos trabalhadores ${ }^{(15-19)}$. O excesso de demanda atrapalha o fluxo da UBS e as rotinas, compromete o acompanhamento de grupos prioritários e o planejamento das equipes ${ }^{(19)}$.

Os demais motivos que aumentam as cargas de trabalho psíquicas são relacionados à insatisfação dos usuários devido à complexidade das suas necessidades e ao desconhecimento do modelo ESF/APS, o que muitas vezes leva à busca por condutas tipicamente biomédicas e curativistas. Essa situação gera insatisfação nos usuários, à medida que seus anseios por uma assistência rápida e curativa não são alcançados. Ações de promoção e prevenção, vínculo e longitudinalidade são difíceis de efetivar, afetando negativamente as relações entre usuários e profissionais ${ }^{(12)}$. Usuários insatisfeitos pressionam e/ou ofendem os profissionais, fato que causa sofrimento psíquico, desmotivação e estresse ${ }^{(19)}$. Na perspectiva inversa ${ }^{(20)}$, ressalta-se a importância da ampliação da prática clínica dos enfermeiros na APS, que resulta em melhor acesso aos serviços. Consultas com maior tempo de duração e comunicação mais eficiente contribuem para a melhora na adesão ao tratamento. Para os enfermeiros, a prática clínica contribui para a maior autonomia e reconhecimento, bem como para a ampliação do corpo de conhecimentos da profissão ${ }^{(20)}$.

As cargas de trabalho psíquicas foram igualmente mencionadas por enfermeiros, técnicos e auxiliares de enfermagem nas cinco regiões. Contudo, a sobrecarga de atividades dos enfermeiros esteve mais relacionada ao acúmulo de atividades educativas, assistenciais e gerenciais ${ }^{(16)}$, enquanto a dos técnicos e auxiliares de enfermagem teve associação com a realização de diversas atividades concomitantemente, em razão da falta de profissionais, fazendo com que assumissem o trabalho de colegas. Essa realidade pode elevar o desgaste, como mencionado em outros estudos ${ }^{(18,21)}$. Dessa forma, pode reduzir a qualidade do cuidado prestado e, consequentemente, o potencial de resolutividade dos serviços de saúde e o acesso dos usuários aos serviços ${ }^{(22)}$.

As cargas de trabalho fisiológicas mostraram-se decorrentes dos problemas gerenciais, como o excesso de demanda, o número insuficiente de funcionários e a jornada de trabalho excessiva, tendo como consequência o desgaste físico acentuado, por vezes materializado em cansaço físico, obesidade, hipertensão arterial sistêmica, dores físicas, dentre outros. As posições incômodas, os movimentos repetitivos e os esforços físicos decorrem da superutilização da força de trabalho pela escassez de trabalhadores ${ }^{(23)}$, sendo mais mencionadas por técnicos e auxiliares de enfermagem pelo contato direto com o usuário.

A estrutura física emergiu como inadequada, elevando a carga biológica, a exemplo da umidade que favorece o surgimento de leveduras (mofo) e insetos, pouca ventilação e luminosidade. Os auxiliares e técnicos de enfermagem estão mais expostos a esse tipo de carga pelo contato próximo com o usuário e pela realização de procedimentos técnicos ${ }^{(18,24)}$. 
Por possuírem materialidade externa ao corpo do trabalhador, as cargas de trabalho físicas, mecânicas e químicas podem ser observadas mais facilmente ${ }^{(9)}$. Estão fortemente associadas ao ambiente de trabalho e problemas estruturais, como ausência ou tamanho insuficiente de espaços para atendimento ao usuário, equipamentos inadequados, estruturas insalubres, exposição à umidade, ruído e calor decorrente da falta de climatização.

Quanto às fontes de redução das cargas de trabalho da enfermagem, a divisão e o planejamento das ações em equipe constituíram-se como mais significativas, caracterizadas pela cooperação e corresponsabilidade na equipe de enfermagem em processos decisórios e de cuidado. $\mathrm{O}$ bom relacionamento na equipe contribui para um trabalho mais prazeroso e menos desgastante. Para isso, é necessário o apoio dos gestores e demais membros das equipes, a valorização das decisões coletivas e o fortalecimento da organização do trabalho interprofissional ${ }^{(7)}$.

A afinidade com os atributos prescritos no modelo assistencial da ESF/APS, como o trabalho em equipe, a dimensão preventiva e a longitudinalidade do cuidado, além do vínculo terapêutico com o usuário alcançado por meio da escuta e do acolhimento, é importante ${ }^{(25)}$ e contribui para a redução das cargas de trabalho de quem atua nesse cenário. Conhecer a realidade dos usuários, a história de vida e o contexto social em que estão inseridos produz na categoria efeito positivo e redutor, favorecendo a longitudinalidade do cuidado e a criação de estratégias coletivas e individuais, de acordo com a realidade do território.

O reconhecimento do trabalho realizado, a disponibilidade de recursos físicos e materiais e de força de trabalho adequados favorece a resolubilidade da assistência, reduzindo as cargas de trabalho dos profissionais de enfermagem.

Outros autores ${ }^{(25-26)}$ defendem que se deve investir na melhoria da avaliação e no acompanhamento do desempenho das organizações, com vistas à excelência da qualidade assistencial. Para isso, é necessário focar na qualificação dos profissionais e na análise do contexto sociopolítico, econômico e de saúde.

A enfermagem é responsável por inúmeras atividades na ESF, de modo que realizá-las em boas condições e ambientes adequados pode contribuir para a ampliação do acesso e resolubilidade da assistência. Cenários positivos e favoráveis reduzem as cargas de trabalho de profissionais e promovem a satisfação de profissionais e usuários.

Como limite do estudo, destaca-se a temporalidade da coleta de dados que, apesar de necessária para a realização de um estudo em profundidade em cenário de vasta dimensão territorial, dificulta a análise das implicações de mudanças macropolíticas na prática, o que poderia ser apreendido em estudos longitudinais.

\section{CONCLUSÃO}

Identificou-se no estudo com profissionais de enfermagem da equipe de Saúde da Família, em diferentes cenários do país, que as cargas de trabalho psíquicas são responsáveis pelo maior desgaste dos trabalhadores da categoria, materializado em processos corporais alterados, como insônia e ansiedade, dentre outros. As demais cargas também afetam o conjunto da equipe de enfermagem, interatuando entre si e com o corpo do trabalhador, o que potencializa o desgaste físico e, especialmente, o psíquico. As cargas químicas foram pouco reconhecidas pelos profissionais nos cenários pesquisados.

O estudo foi intencionalmente definido para analisar o fenômeno em cenários reconhecidos como de boa qualidade. No entanto, os resultados encontrados mostram que os trabalhadores de enfermagem que atuam na ESF no Brasil estão expostos a cargas de trabalho geradas principalmente por problemas estruturais relacionados à precariedade das condições de trabalho e à sobrecarga.Tal dado sinaliza uma realidade mais dramática, que poderá ser evidenciada em outros estudos, assim como apresenta um desafio para gestores, estudiosos, trabalhadores da saúde e legisladores no sentido de enfrentamento dos problemas identificados.

Sugere-se, para reduzir as cargas de trabalho, priorizar o olhar para a força de trabalho da enfermagem, investindo na valorização do trabalho desenvolvido por esses profissionais e no provimento de meios de trabalho que possibilitem uma assistência mais resolutiva e menos desgastante.

\section{RESUMO}

Objetivo: Identificar as cargas de trabalho de equipes de enfermagem que atuam na Estratégia Saúde da Família nas cinco regiões do Brasil e as interfaces com o desgaste desses profissionais. Método: Estudo qualitativo com profissionais de enfermagem de 20 Unidades de Saúde da Família, de oito munícipios das cinco regiões do país. Os dados foram coletados por meio de entrevistas e observação, sendo analisados segundo preceitos da análise temática e do referencial teórico de cargas de trabalho. Resultados: Participaram 79 profissionais. As cargas de trabalho foram relacionadas a problemas estruturais e gerenciais, destacando-se as psíquicas devido ao excesso de demanda e à falta de profissionais, as fisiológicas pela sobrecarga de atividades que geram dores físicas e exaustão, as físicas e mecânicas em função das inadequações no ambiente de trabalho e equipamentos, as biológicas pela presença de micro-organismos e as químicas devido à exposição a poeiras e fumaças. Conclusão: As fontes que elevam as cargas de trabalho são semelhantes nas cinco regiões, indicando forte influência das cargas psíquicas e fisiológicas nos padrões de desgaste da enfermagem. Entretanto, divisão e planejamento das ações em equipe e afinidade com o modelo assistencial reduzem as cargas de trabalho.

\section{DESCRITORES}

Enfermagem de Atenção Primária; Carga de Trabalho; Equipe de Enfermagem; Saúde do Trabalhador; Estratégia Saúde da Família.

\section{RESUMEN}

Objetivo: Identificar las cargas de trabajo de los equipos de enfermería de la Estrategia de Salud Familiar en las cinco regiones de Brasil y las interfaces con el desgaste de estos profesionales. Método: Se trata de un estudio cualitativo realizado entre 79 profesionales de 
enfermería de veinte Unidades de Salud Familiar, en ocho municipios de las cinco regiones del país. Los datos se recogieron mediante entrevistas y observación y se analizaron de acuerdo con los preceptos del análisis temático y de la referencia teórica de las cargas de trabajo. Resultados: Las cargas de trabajo estaban relacionadas con problemas estructurales y de gestión, destacándose las psíquicas por el exceso de demanda y la falta de profesionales; las fisiológicas por la sobrecarga de actividades que generan dolor físico y agotamiento; las físicas y mecánicas por las inadecuaciones en el ambiente de trabajo y los equipos; las biológicas por la presencia de microorganismos y las químicas por la exposición al polvo y al humo. Conclusión: Las fuentes que aumentan las cargas de trabajo son similares en las cinco regiones, lo que indica una gran influencia de las cargas psíquicas y fisiológicas en los patrones de desgaste de la enfermería. Sin embargo, la división y la planificación de las acciones en equipo y la afinidad con el modelo de atención reducen las cargas de trabajo.

\section{DESCRIPTORES}

Enfermería de Atención Primaria; Carga de Trabajo; Grupo de Enfermería; Salud Laboral; Estrategia de Salud Familiar.

\section{REFERÊNCIAS}

1. Brasil. Ministério da Saúde. Portaria n. 2.436. Aprova a Política Nacional de Atenção Básica, estabelecendo a revisão de diretrizes para a organização da Atenção Básica, no âmbito do Sistema Único de Saúde [Internet]. Brasília; 2017 [citado 2018 mar. 13]. Disponível em: http://bvsms.saude.gov.br/bvs/saudelegis/gm/2017/prt2436_22_09_2017.html

2. Malta DC, Santos MAS, Stopa SR, Vieira JEB, Melo EA, Reis AAC. Family Health Strategy Coverage in Brazil, according to the National Health Survey, 2013. Ciênc Saúde Coletiva. 2016;21(2):327-38. DOI: 10.1590/1413-81232015212.23602015.

3. Thum E, Fehn AC, Acioli S, Fassa MEG. Formação e prática de enfermeiros para a Atenção Primária à Saúde - avanços, desafios e estratégias para fortalecimento do Sistema Único de Saúde. Saúde Debate. 2018;42(1):275-88. DOI: 10.1590/0103-11042018s118

4. Swan M, Ferguson S, Chang A, Larson E, Smaldone A. Quality of primary care by advanced practice nurses: a systematic review. Int J Qual Health Care. 2015;27(5):396-404. DOI: 10.1093/intqhc/mzv054

5. Cassiani SHB. Strategy for universal access to health and universal health coverage and the contribution of the International Nursing Networks. Rev Latino Am Enfermagem. 2014;22(6):891-2. DOI: 10.1590/0104-1169.0000.2502.

6. Grant J, Lines L, Darbyshire P, Parry Y. How do nurse practitioners work in primary health care settings? A scoping review. Int J Nurs Stud. 2017;75:51-7. DOI: 10.1016/j.ijnurstu.2017.06.011.

7. Ammi M, Ambrose S, Hogg B, Wong S. The influence of registered nurses and nurse practitioners on patient experience with primary care: results from the Canadian QUALICO-PC study. Health Policy. 2017;121(12):1215-24. DOI: 10.1016/j.healthpol.2017.09.019

8. GBD 2016 Healthcare Access and Quality Collaborators. Measuring performance on the Healthcare Access and Quality Index for 195 countries and territories and selected subnational locations: a systematic analysis from the Global Burden of Disease Study 2016. Lancet. 2018;391:2236-71. DOI: 10.1016/S0140-6736(18)30994-2.

9. Laurell AC, Noriega M. Processo de produção e saúde: trabalho e desgaste operário. São Paulo: Hucitec; 1989.

10. Brasil. Ministério da Saúde. Portaria $\mathrm{n}^{\circ}$ 1.645, de 2 de outubro de 2015. Dispõe sobre o Programa Nacional de Melhoria do Acesso e da Qualidade da Atenção Básica (PMAQ-AB) [Internet]. Brasília; 2015 [citado 2018 mar. 13]. Disponível em: http://bvsms.saude.gov.br/bvs/ saudelegis/gm/2015/prt1645_01_10_2015.html

11. Machado MH, Filho WA, Lacerda WF, Oliveira E, Lemos W, Wermelinger M, et al. Características gerais da enfermagem: o perfil sócio demográfico. Enferm Foco. 2016;6(1-4):11-7. DOI: 10.21675/2357-707X.2016.v7.nESP.686.

12. Faraz A. Novice nurse practitioner workforce transition and turnover intention in primary care. J Am Assoc Nurse Pract. 2017;29(1):26-34. DOI 10.1002/2327-6924.12381.

13. Aiken LH, Sloane DM, Bruyneel L, Van den Heede K, Griffiths P, Busse R, et al. Nurse staffing and education and hospital mortality in nine European countries: a retrospective observational study. Lancet. 2014;24;383(9931):1824-30. DOI: 10.1016/S0140-6736(13)62631-8.

14. Vilela EN, Mafra LA. Estratégia Saúde da Família: contratação temporária e precarização nas relações de trabalho. Cad Estudos Interdisc [Internet]. 2015 [citado 2018 mar. 25]. Disponível em: https://publicacoes.unifal-mg.edu.br/re-vistas/index.php/cei/article/view/410/pdf.

15. Biff D, Pires DEP, Machado FL, Oliveira JSA, Souza DF. Workloads of nurses in the Family Health in Brazil: what the literature says. Int Arch Med. 2016;9. DOI: http://dx.doi.org/10.3823/2187

16. Ferreira SRS, Perico LAD, Dias VRFG. The complexity of the work of nurses in Primary Health Care. Rev Bras Enferm. 2018;71 Suppl 1:704-709. DOI: 10.1590/0034-7167-2017-0471.

17. Facchini LA, Tomasi E, Diélio AS. Quality of Primary Health Care in Brazil: advances, challenges and perspectives. Saúde Debate. 2018;42(1):208-223. DOI: 10.1590/0103-11042018S114

18. Scherer MDA, Oliveira NA, Pires DEP, Trindade LL, Gonçalves ASR, Vieira M. Increased workloads for nurse technicians in primary health care in Brazil. Trab Educ Saúde. 2016;14 Suppl 1:89-104. DOI: 10.1590/1981-7746-sol00030

19. Trifunovic N, Jatic Z, Kulenovic AD. Identification of causes of the occupational stress for health providers at different levels of health care. Med Arch. 2017;71(3):169-72. DOI: 10.5455/medarh.2017.71.169-172

20. Toso BRGO, Filippon J, Giovanella L. Nurses' performance on primary care in the National Health Service in England. Rev Bras Enferm. 2016;69(1):182-91. DOI: 10.1590/0034-7167.2016690124i

21. Soratto J, Pires DEP, Trindade LL, Oliveira JSA, Forte ECN, Melo TP. Job dissatisfaction among health professionals working in the family health strategy. Texto Contexto Enferm. 2017;26(3):1-11. DOI: 10.1590/0104-07072017002500016.

22. Pires DEP, Machado RR, Soratto J, Scherer MA, Gonçalves ASR, Trindade LL. Nursing workloads in family health: implications for universal access. Rev Latino Am Enfermagem. 2016;24:e2682. DOI: 10.1590/1518-8345.0992.2682.

23. Felli VEA, Costa TF, Baptista PCP, Guimarães ALO, Anginoni BM. Exposure of nursing workers to workloads and their consequences. Rev Esc Enferm USP . 2015;49(n.spe2):98-105. DOI: 10.1590/S0080-623420150000800014. 
24. Freire MN, Costa ER. Qualidade de vida dos profissionais de enfermagem no ambiente de trabalho. Rev Enferm Cont. 2016;5(1):151-8. DOI: $10.17267 / 2317-3378$ rec.v5i1.871.

25. Costa C, Laimer VR, Piovesan RTC, Coelho EA. Competências gerenciais importantes em uma organização hospitalar. RAIMED - Revista de Administração IMED. 2016;6:45-55. DOI: 10.18256/2237-7956/raimed.v6n1p45-55

26. Camelo SHH, Soares MI, Chaves LDP, Rocha FLR, Silva VLS. Nurse managers at a teaching hospital: training, responsibilities and challenges. Rev Enferm UERJ. 2016;24:1-6. DOI: 10.12957/reuerj.2016.11637.

\section{Apoio financeiro}

Conselho Nacional de Desenvolvimento Científico e Tecnológico (CNPq). Processo 402871/2016-2. Edital Universal e 303469/2014-5 Projeto Bolsa PQ. 